\title{
Feasibility Study of a Combined Tension-Torsion Hopkinson Bar
}

\author{
Marco Sasso ${ }^{1 *}$, Edoardo Mancini ${ }^{2}$ \\ ${ }^{1}$ Università Politecnica delle Marche - DIISM, via Brecce Bianche, 60131, Ancona, Italy \\ ${ }^{2}$ Università degli Studi dell'Aquila, Piazzale E. Pontieri, 67100, L'Aquila, Italy
}

\begin{abstract}
The increasing interest to improve the description of the plastic behavior and the fracture prediction for ductile materials under complex loading conditions conducted the researchers to overcome the $\mathrm{J} 2$ plasticity theory. To do this more sophisticated plasticity model base on ductile damage have been implemented. Material model parameters must be identified by means of proper testing and calibration procedures requiring different loading conditions. Different types of tests must be performed, imposing multiaxial stress paths to the specimens. Tensile tests on smooth and round notched bars, plane strain tests, torsion tests, compression and combined tension-torsion tests on hollow and solid cylindrical bars must be executed. While multiple works are present in literature for model assessment and validation in quasi-static conditions, nothing can be found at high strain rate in biaxial conditions (tension-torsion). Biaxial tests in dynamic conditions are very difficult to carry out especially if you are interested to register the entire story of stress and strain. In this work, analytical and numerical study to evaluate the feasibility to carry out dynamic tension, dynamic torsion, dynamic torsion-static tension/compression and dynamic tension-dynamic torsion tests is discussed. The tests will be performed using a properly designed Split Hopkinson Bar.
\end{abstract}

\section{Introduction}

Considering high strain rate testing, the most commonly used apparatus for tensioncompression and torsional loading are the split Hopkinson tensile bar [1] and torsional split Hopkinson bar [2]. The last one is of interest to obtain very large strains. Indeed, the strains that can be achieved by tension is limited by necking and compression is limited by barrelling of the specimen, moreover, additional drawbacks arise from the dispersion of longitudinal stress wave as it travels along the elastic bars in a compression or tension test are not present in a torsion test. Therefore, many are the advantages to obtaining the stress-strain behaviour by the torsional test.

Furthermore, the increasing interest to improve the description of the plastic behaviour and the fracture prediction for ductile materials lead to imposing multiaxial stress paths to the specimens. However, it is very difficult to carry out tests in dynamic conditions and

\footnotetext{
* Corresponding author: $\underline{\text { m.sasso@staff.univpm.it }}$
} 
multiaxial stress state with a unique apparatus, especially if the recording of the entire stress and strain history is of interest.

To the authors knowledge, combined tension-torsion tests are conducted only on static machines; no dynamic tension-torsion facility and data are available in the literature. The objective of this paper is to reveal the feasibility study and the subsequent design of an apparatus able to carry out simultaneous dynamic tension-torsion test by Split Hopkinson bar technique. The waves propagation has been studied analytically by Lagrangian diagram and verified by a finite element simulation. The preliminary design of the apparatus has been developed, which allows for twist angle in the order of 360 degree and $50 \mathrm{~mm}$ of longitudinal displacement.

\section{Apparatus solution}

The general design of the proposed Split Hopkinson Tension Torsion Bar (SHTTB) is similar to the direct tension-compression Hopkinson bar already developed by the authors [3], and is based on pre-stressing a portion of the incident bar, as implemented in [4-6]. The SHTTB is made of several aligned metallic bars, two static clamps, a loading system to twist the first bar and to pull the first and the second bars simultaneously and two end arrests. The first two bars represent the pre-stressed (analogous to the striker bar in convention SHPB), whereas the other bars form the input and output bars. The sample is placed between the input and output bars.

The clamps are used to prevent the bar from rotating and translating, while torque and tensile loads are applied by a loading mechanism at the first end of the pre-stressed bars. The pre-stressed bar pre-loaded in tension and torsion is guided and held adjacent to input end of the input bar (and remains adjacent the input bar during application of the pre-load force) by a collar; indeed, it is very important to preserve the mechanical continuity between the bars.

The sudden failure of a sacrificial element releases the torque and traction that are stored between the loading end of the pre-stressed bar and the first and second clamps; this generates a shear wave and a compressive wave that propagate through the input bar. It is well known that the longitudinal wave travels at higher speed than the torsional wave; hence, the longitudinal wave is made to travel for a longer distance, i.e. it is made to reach the output bar (without stressing the sample) and is reflected back at the free end as a tensile wave. The latter travels back through the output bar and arrives at the sample location for the "rendezvous" with the torsion wave that is still coming from the input bar.

The principle of simultaneous generation of normal and shear stress pulses that are made to reach the sample at the same instant exploiting the different travel lengths is currently applied for international patent.

A schematic diagram of the apparatus is shown in Figure 1. The SHTTB consists of fifteen titanium alloy bars (Ti-4Al-6V), all of $20 \mathrm{~mm}$ diameter and approximately $6 \mathrm{~m}$ in length. The choice of adopting such a considerable length was dictated by the intention of performing tests up to failure on ductile metals, which may require very large rotations and displacements at the sample interfaces.

\subsection{Waves generation}

The tension and torsion waves are generated by two electromechanical actuators connected in series, as shown in figure $2 \mathrm{a}$, the former being a linear actuator, the latter being a worm gearmotor. 


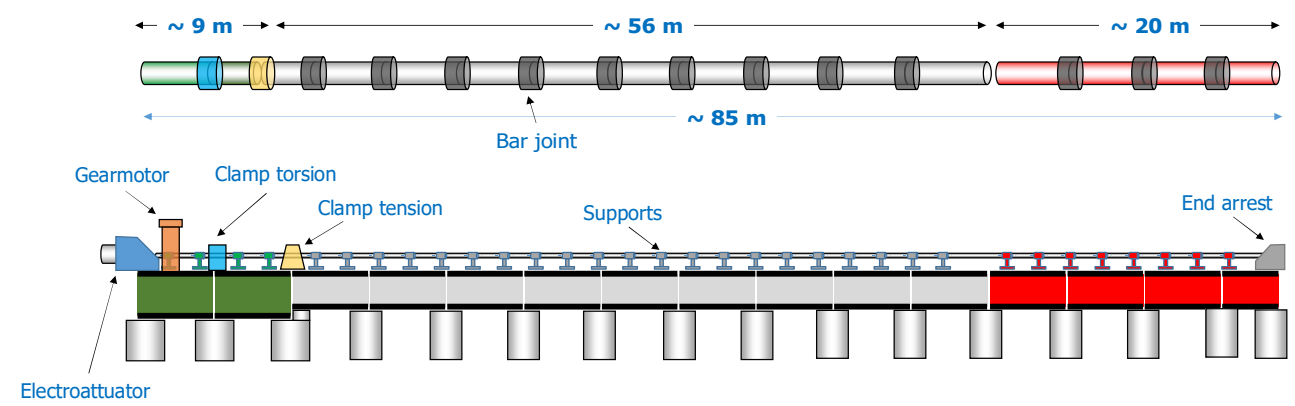

Fig. 1. Scheme of the Tensional-Torsional Hopkinson bar

Hence, two distinct clamps are needed, as shown in Figure 2b; a former one is needed to contrast the torque, a latter one, which is adjacent to the input bar, is needed to contrast the axial load. Moreover, the two actuators are independently operated, so that any combination of static tension/torsion pre-loads can be applied. As aforementioned, the waves' generation is triggered by the brittle failure of a sacrificial element that resists both axial and torsional static preload, until its maximum strength is exceeded by the applied forces.

a)

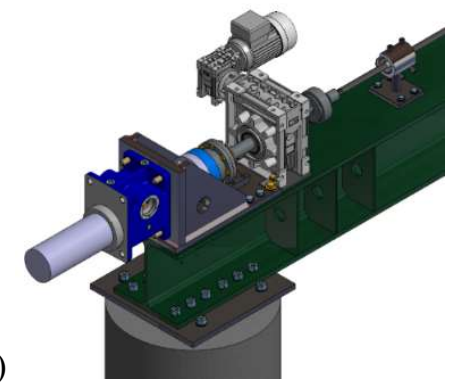

b)

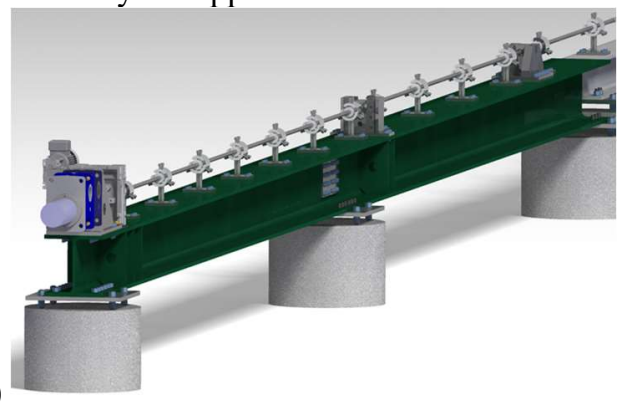

Fig. 2. a) loading system, b) pre-stressed bar with double clamping system

Multiple test types can be performed, depending on how the preload is applied and eventually released: sudden release of a static pre-tension generates a compression wave that can be used for compression and indirect tension tests (see Figure $3 \mathrm{a}$ and $3 \mathrm{~b}$ ). Note that the traveling wave has half intensity but double spatial length with respect to the pre-stressed bar length. If no axial load is provided, pure dynamic torsion is achieved (Figure 3c). The application of static compression or tension to the whole system allows for dynamic biaxial tests, where the dynamic torsion is superimposed to a static stress (Figure $3 \mathrm{~d}$ and $3 \mathrm{e}$ ). In such a case, a third clamp is needed at the end of the output bar; obviously, the condition expressed in Figure $3 \mathrm{~d}$ could suffer of limitations from the risk of buckling. Finally, the most interesting arrangement consists in static pre-tension and pre-torsion that are applied to the pre-stressed bar and released simultaneously; in this way simultaneous dynamic tension and torsion tests can be performed on the sample placed between the input and output bar (Figure $3 \mathrm{f}$ ).

In view of the last configuration, the pre-stressed bar could be statically loaded in tension and torsion for its whole length; however, if the same time duration is desired for the longitudinal and torsional waves, only a portion of the pre-stressed bar should be statically loaded in torsion. In such a case, the length of the pre-stressed bar that is statically twisted, $L_{P T}$, and the length of the entire pre-stressed bar that is statically loaded in tension, $L_{P L}$, are related by $L_{P T} / L_{P L}=C_{T} / C_{L}$, where $C_{T}$ and $C_{L}$ are the propagation speeds of torsion and 
longitudinal waves, respectively. They are given by $C_{T}=(G / \rho)^{0.5}$ and $C_{L}=(E / \rho)^{0.5}$, where $E$ and $G$ are the normal and shear elastic modulus, and $\rho$ is the density of the bars' material.

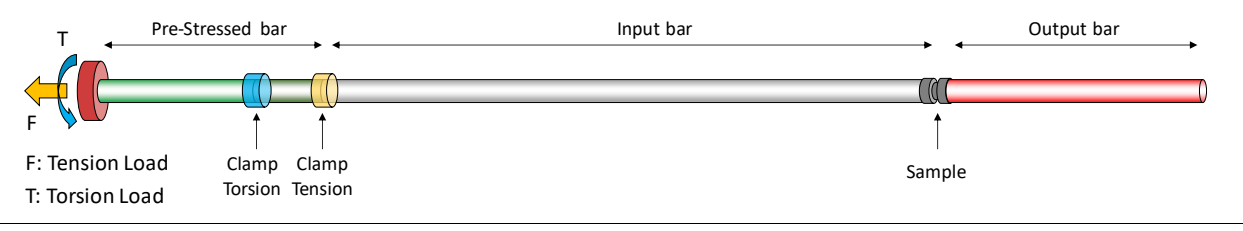

(a) Dynamic Compression

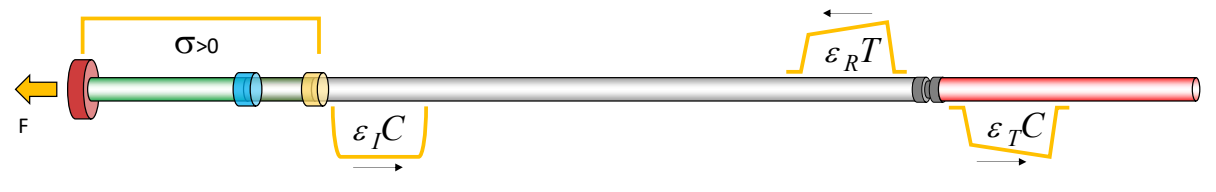

(b) Indirect Dynamic Tension

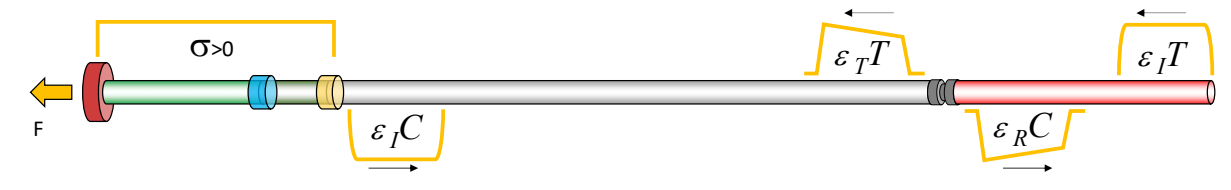

(c) Dynamic Torsion

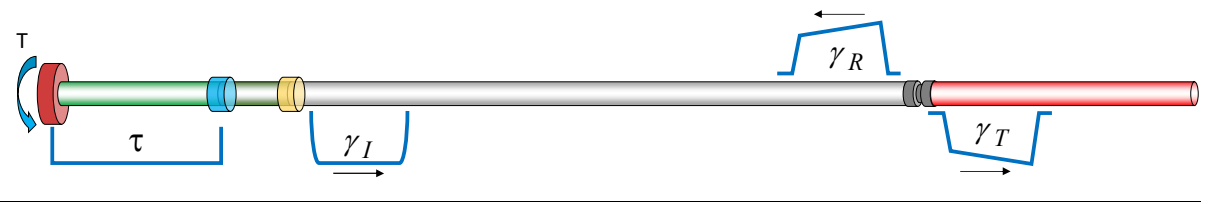

(d) Dynamic Torsion - Static Compression

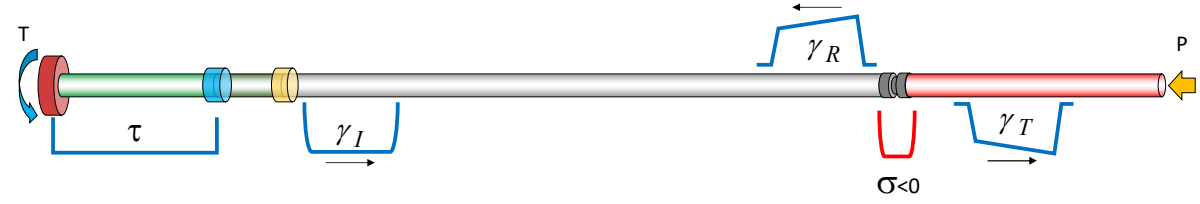

(e) Dynamic Torsion - Static Tension

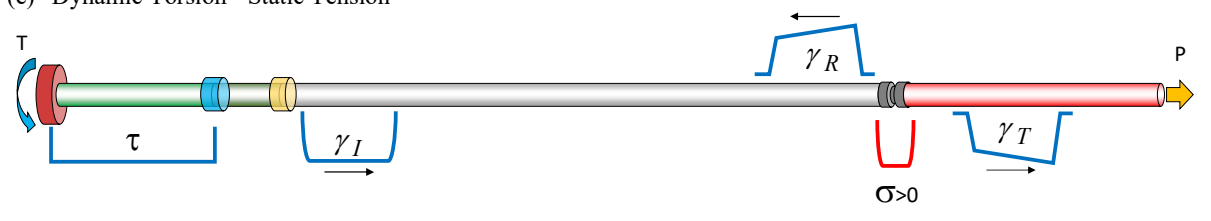

(f) Dynamic Tension - Dynamic Torsion

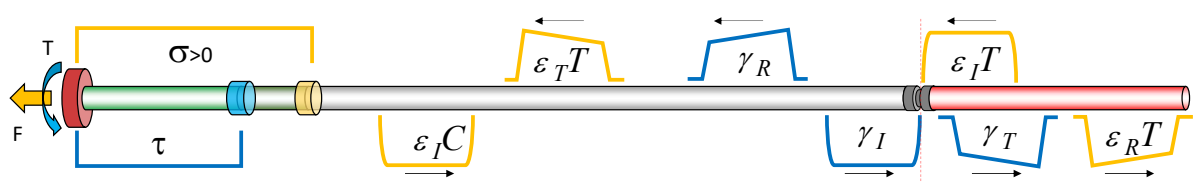

Fig. 3. Wave generation: schematic representation of direct tension-torsion apparatus; $\varepsilon_{\mathrm{IC}}$ : compression incident wave, हाт: tension incident wave, єтт: tension transmitted wave, हRт: tension reflected wave, $\gamma \mathrm{I}$ : torsional incident wave, $\gamma_{T}$ : torsional transmitted wave, $\gamma_{\mathrm{R}}$ : torsional reflected wave 


\section{TENSION-TORSION WAVES SYNCHRONIZATION AND LAGRANGE DIAGRAM}

\subsection{Tension-Torsion waves synchronization}

Considering a system of long aligned rods, tension and compression waves propagate at the same speed, whereas the torsion waves propagate at a significantly different one, about $60 \%$ of the speed of the tension / compression waves, depending on the exact value of Poisson ratio.

This means that if one desires to use such waves to load dynamically a sample sandwiched between two bars, the different types of waves should be generated at different locations and/or different instants in a combination that makes them to arrive at the sample location simultaneously. The methods commonly used for generating these waves are based on impacts or on sudden load release (e.g. by a rapid unclamp or a brittle failure of some connecting element); however, the synchronization of such events is very difficult, not to say impossible, to accomplish if the desired accuracy is in the order of a few $\mu s$.

The idea proposed in this work consists in generating the two waves at the same instant $\mathrm{t}_{0}$, and make them propagate for two different distances; the scheme of the indirect tension Hopkinson bar is used. After pre-loading the pre-stressed bar in both tension and torsion, the failure of a sacrificial element that connect the first end of the pre-stressed bar to the actuating system, as in [3], will result in a compression and torsion stress wave of a precisely known amplitude and duration that propagate through the input bar. The compression wave reaches the specimen area first. A special design let the compression wave pass into the output bar without stressing the sample: to this purpose, an external collar could be used with solid cylindrical samples, whereas for hollow samples, it will be sufficient to put the input and output bar into contact. Then, as in the scheme of indirect tension Hopkinson bars, the wave is reflected as a tensile wave at the free end of the output bar.

\subsection{Lagrangian diagram}

The Lagrangian space-time diagram of the system is shown in Figure 4. The position of the shear and normal front waves as a function of time can be obtained by this diagram. It is possible to see that, upon load release, half of the stored axial force and torque will travel as elastic axial stress and shear waves, respectively, towards the input bar and the sample at the longitudinal $\left(C_{L}\right)$ and shear $\left(C_{T}\right)$ waves speed. Normal and shear pulse of the same magnitude will travel at the proper speed in the other direction unloading the force and torque that are stored in the pre-stressed bar. These unloading pulses will reflect completely at the loading system and travel toward the specimen releasing the axial force and torque to zero; therefore, the total length of the normal and shear pulses that will pull and twist the specimen are equal to the time taken by the unloading pulses to travel toward the loading system and back to the torsion and tension clamps. In general by relocating the clamps (the same is obtained by replacing the pre-stressed bar with one of different length [7]) the pulses length can be changed.

The pre-stressed, input and output bars are in such length ratio that the tensile wave reaches the specimen at the same moment of the generated torsion wave. As a result, a configuration has been conceived where the pre-stressed, input and output bar are 9, 56 and $20 \mathrm{~m}$ long, respectively. All bars are in grade 5 titanium of $20 \mathrm{~mm}$ diameter. By applying 100 $\mathrm{kN}$ and $350 \mathrm{Nm}$ of static preload, the maximum available stroke at the input/output bar interface will be in the order of $50 \mathrm{~mm}$ of elongation $2 \pi$ rotation. 


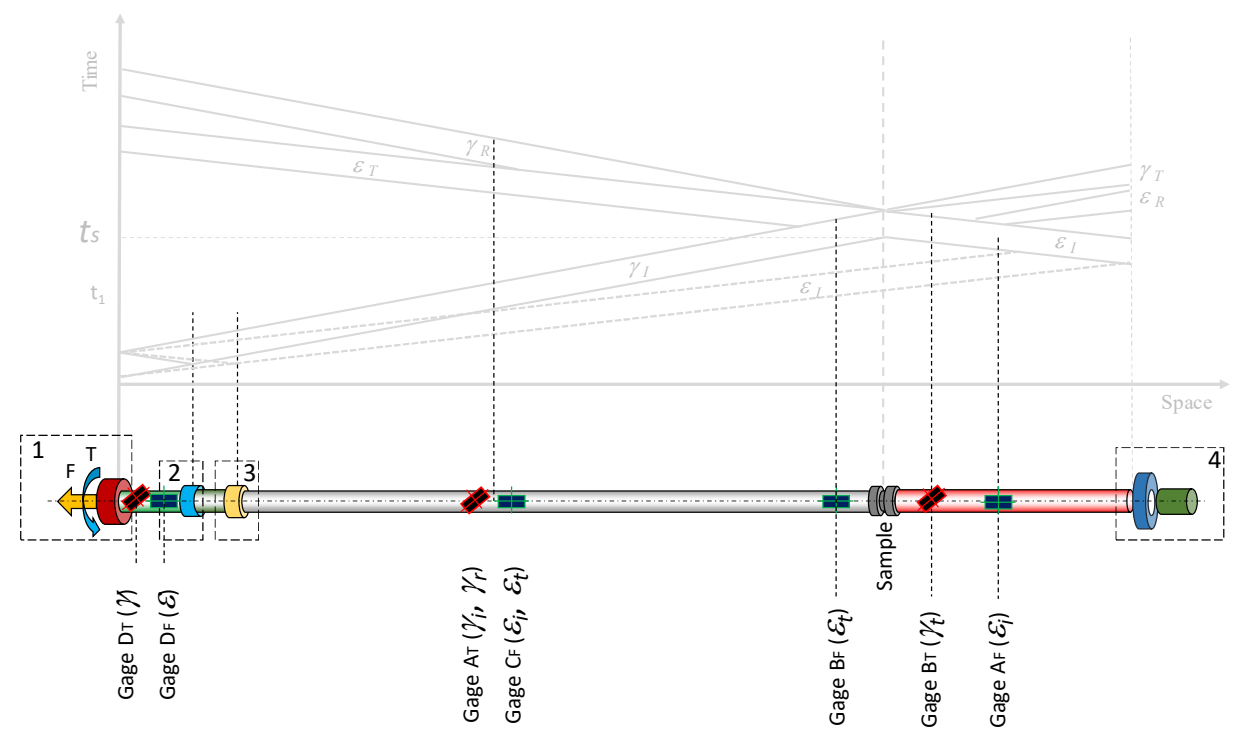

Fig. 4. Lagrangian diagram of the whole apparatus.

The Lagrange diagram can be used to select the most suitable locations for installing the strain gauges on the input and output bars, in order to measure the stress and strain experienced by the sample. There are no significant issues in the positioning the strain gauges to measure the transmitted pulses, as they can be put on the bars close to the specimen interface; differently, the reading of the reflected longitudinal wave requires the strain gage $A_{F}$ to be placed at half length of the output bar with limited possible changes. However, it must be admitted that with the proposed configuration, where several bars are connected, it will be preferable to use high speed imaging technique to measure the strain directly on the sample.

\section{Numerical verification}

Numerical simulations have been performed on a FE model with an explicit solver in order to reproduce the wave travelling and the deformation of a hypothetical sample. A 3D model has been necessary because of no symmetry can be considered due to simultaneously application of tension and torsion load; 8 node BRICK elements have been used. Some details of the model are shown in Figure 5. The torsion and tension pulse were generated by assigning a displacement and a rotation at the first end of the pre-stressed bar, and then release the constraint. The imposed constraints correspond to approximately $100 \mathrm{kN}$ and $300 \mathrm{Nm}$. Rigid walls has been used to model clamps 2 and 3.

All the required contacts constraints were modelled as automatic surface-to-surface contact, based on the penalty stiffness algorithm. The connection between clamps and bars were modelled by node merge.

The adopted specimen geometry is inspired to the shape proposed in [8]. It consists of hollow tubular sample with a thread and a groove which transmit the tension and torsion loads. The inner diameter of hollow sample is accommodate the input and output bar extremities which are in contact, permitting the first compression wave to pass without deforming the sample significantly. The material choose is an AA7075 state O modelled with Johnson-Cook's law [9]. 


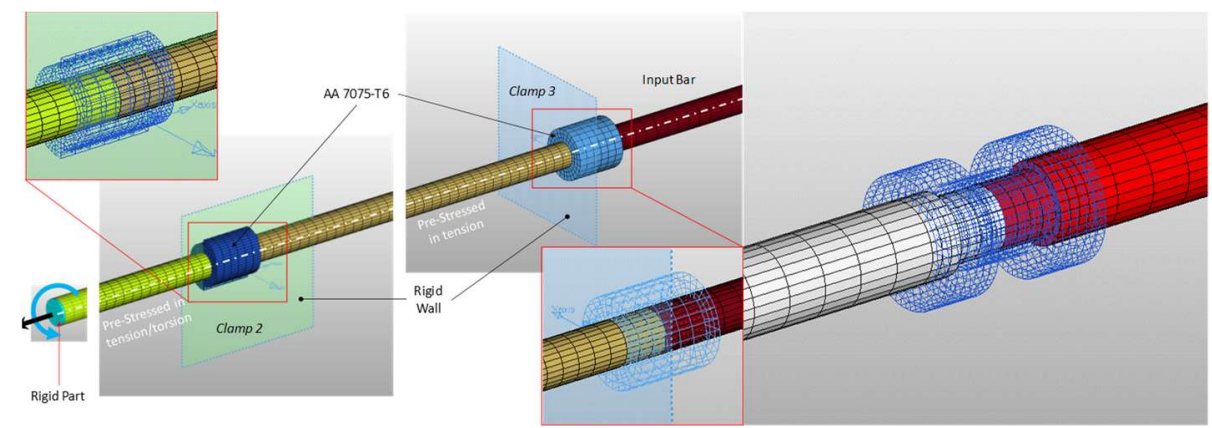

Fig. 5. FEM model of the SHTT bar

Figure 6 reports pictures of the sample captured at three instant of time. The sample is in wireframe visualization while the bars are shaded. The first compression wave transits across the sample without deforming it (Figure 6b); the sample gage length is verified to by unchanged with respect to the undeformed configuration (Figure 6a). Successively, the longitudinal wave (which has been reflected into tension by the free end of the output bar) and the torsional wave deform the specimen simultaneously, while input and output bars are detached (Figure 6c).

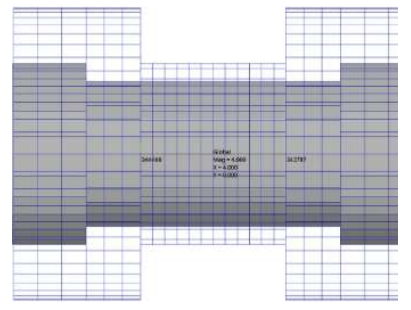

(a)

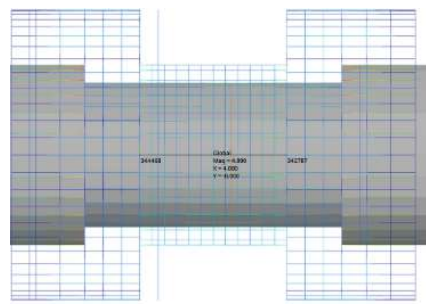

(b)

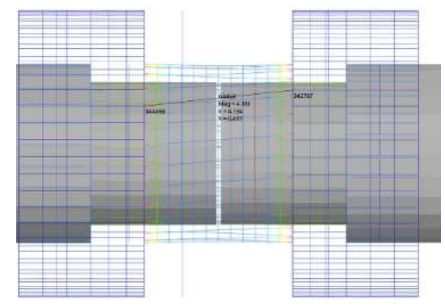

(c)

Fig. 6: Three instants of time: (a) @start of the analysis, (b) after compression wave transit and (c) after simultaneous tension and torsion waves transit

Figure 7 shows the signal recorded by two virtual strain gauges placed near the sample in the input and output bar. A perfect synchronization between two waves can be achieved.

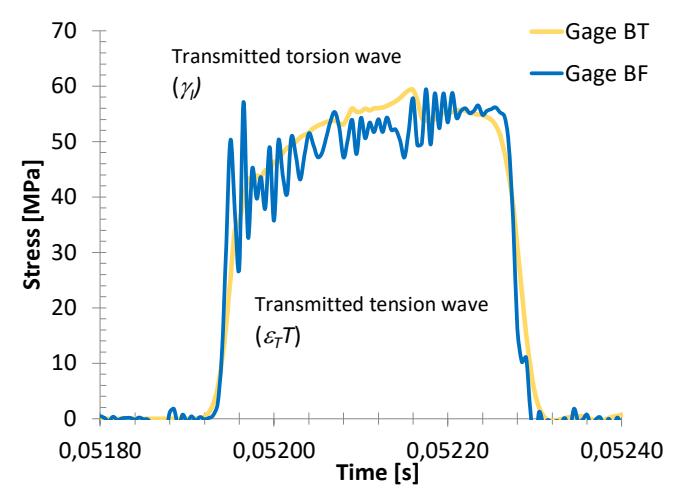

Fig. 7. Waves recorded by the virtual strain gauges in position $\mathrm{B}_{\mathrm{T}}$ and $\mathrm{B}_{\mathrm{F}}$ 


\section{Conclusions}

In this work a solution for performing simultaneous tension-torsion tests on materials at high strain rate is proposed. A compression and a torsion wave are concurrently generated at the end of the pre-stressed bar by the failure of a fragile element. The compressive wave, which propagates at higher speed, is made to travel up to the end of the output bar, where it is reflected back into tension wave; then, by properly tuning the length of the input and output bars, the tensile and the torsion waves deform the sample simultaneously. A very long configuration has been designed, i.e. $9 \mathrm{~m}, 56 \mathrm{~m}$ and $20 \mathrm{~m}$ for the pre-stressed, input and output bars, respectively; the use of titanium bars will permit to deform ductile metals up to high strains, in both elongation and rotation. The Lagrange diagram also permits to find the correct position of strain gauges for measuring the travelling waves.

\section{Patent application}

The concept of this innovative apparatus has been submitted for international patent application.

\section{References}

1. W. Chen, B. Song, Split Hopkinson (Kolsky) Bar, (Springer, 2011)

2. X. Yu, L. Chen, Q. Fang, X. Jiang, Y. Zhou, Adv. in Civil Eng., 2018, 2719741 (2018)

3. E. Mancini, M. Sasso, M. Rossi, G. Chiappini, G. Newaz, D. Amodio, J. Dyn. Behav. Mater. 1, 201-213 (2015)

4. G.H. Staab, A. Gilat, Exp. Mech. 31, 232-235 (1991)

5. C. Albertini, K. Labises, Split Hopkinson bar testing apparatus, International Patent WO 98/26274 (1998)

6. G. Mirone, Exp. Tech. 39, 3-15 (2015)

7. M. Martarelli, E. Mancini, B. Lonzi, M. Sasso, Meas. Sci. Technol. 29, 025601 (2018)

8. A. Gilat, C.-S. Cheng, Exper. Mech. 40, 54-59 (2002)

9. M. Sasso, A. Forcellese, M. Simoncini, D. Amodio, E. Mancini, High strain rate behaviour of AA7075 aluminum alloy at different initial temper states, KEM 651-653, 114-119 (2015) 